\title{
Epidemiology and Control of Lassa fever
}

\author{
Effiong E Bassey* \\ Department of Applied Microbiology and Brewing, Nnamdi Azikiwe University, Nigeria
}

Received: 阱April 20, 2018; Published: 制 May 01, 2018

*Corresponding author: Effiong E Bassey Department of Applied Microbiology and Brewing, Nnamdi Azikiwe University, Awka, Nigeria

\begin{abstract}
The Lassa Virus (an Arenavirus) is found in West Africa, where it sometimes a severe hemorrhage illness called Lassa fever. The antivirus drug ribavirin is effective, but only if administered early in the course of illness. Because of its expense, need for intravenous administration, potential toxicity, and teratogenicity, empiric therapy with ribavirin is undesirable. Laboratory diagnosis of acute Lassa virus infection has traditionally employed the indirect fluorescent-antibody (IFA) test. However, enzymelinked immunosorbent assays (ELISAs) for Lassa virus antigen and immunoglobin M (IgM and IgG) antibodies have been developed that are thought to be more sensitive and specific likely represent underestimates with. The high sensitive and specificity, capability for early diagnosis, and prognostic value of the ELISAs make them the diagnostic tests of choice for detection of Lassa fever.
\end{abstract}

KeyWords: Epidemiology; Control; Lassa Fever

\section{Introduction}

Lassa fever is a viral hemorrhagic fever transmitted by rats. It has been known since the 1950s, but the virus was not identified until 1969, when two missionary nurses died from it in the town of Lassa in Nigeria. Found predominantly in West Africa, it has the potential to cause tens of thousands of deaths. Even after recovery, the virus remains in body fluids, including semen. The years of civil unrest in Sierra Leone (1991-2002) halted the investigation (through international collaboration) of Lassa fever at a specialist unit Kenema. Increasing international travel and the possibility of use of the Lassa virus as a biological weapon escalate the potential for harm beyond the local level. Access to the country is improving, so renewed efforts to understand it are feasible. The fact that Lassa fever was an arenavirus suggested the existence of a rodent host, which was identified when Lassa virus was isolated from Mastomys natalensis in 1972 [1]. Our knowledge of African arenavirus epidemiology has now been expanded by the isolation of viruses related to Lassa virus from number of rodent species from other parts of Africa (Central Africa Republic, Zimbabwe, Mozambique and South Africa). The relative importance of person to person and rodent-to-person transmission in the natural history of Lassa fever in rural West Africa has not been satisfactorily assessed. Epidemiology studies that would allow independent identification of either mode are difficult to design. The single study thus far attempted indicated that person-to-person spread occurred in villages, but its importance could not be accurately determined [2]. Thus, the epidemiology of Lassa fever has two principal themes: rodent-to human spread, which appear to predominate, and person-to-person spread, which is also of importance.

\section{Epidemiology}

The reservoir or host, of Lassa virus is a rodent known as the "Multimamate rat (Mastomys natalensis). Once infected, this rodent is able to excrete virus in urine for an extended time period, maybe for the rest of its life. Mastomys rodents breed frequently, produce large numbers of offspring, and are numerous in the Savannas and forests of West, central, and east Africa. In addition, Mastomys readily colonize human homes and areas where food is stored. All these factors contribute to the relatively efficient spread of Lassa virus from infected rodents to humans [3].

Transmission of Lassa virus to humans occurs most commonly through ingestion or inhalation. Mastomysrodent shed the virus in urine and dropping and direct contact with these materials through touching soiled objects, eating contaminated food, or exposure to open cuts or sores, can lead to infection [4]. 
Because Mastomys rodents often live in and around homes and scavage on leftover human food items or poorly stored food, direct contact transmission is common. Mastomys rodents are sometimes consumed as a food source and infection may occur when rodents are caught and prepared. Contact with infected rodent excretions. This aerosol or airborne transmission may occur during cleaning activities, such as sweeping.

Direct contact with infected rodents is not the only way in which people are infected; person-to-person transmission may occur after exposure to virus in the blood, tissue, secretions, or excretions of Lassa virus-infected individual. Casual contact (including skinto-skin contact without exchange of body fluids) does not spread Lassa virus. Person-to-person transmission is common in health care [5].

The most common complication of Lassa fever is deafness. Various degrees of deafness occur approximately one-third of infections, and in many cases hearing loss is permanent. As far as is known, severity of the disease does not affect this complication: deafness may develop in mild as well as in severe cases.

Approximately 15\%-20\% of patients hospitalized for Lassa fever die from the illness. However, only $1 \%$ of all Lassa virus infections result in death. The death rates for women in the third trimester of pregnancy are particularly high. Spontaneous abortion is a serious complication of infection with an estimated 95\% mortality in fetuses of infected pregnant mothers. Because the symptoms of Lassa fever are so varied and nonspecific, clinical diagnosis is often difficult. Lassa fever is also associated with occasional epidermics, during which the case-fratality rate can reach $50 \%$ in hospitalized patients.

Symptoms generally appear within 1-3 weeks following infection. An estimated $80 \%$ of infections produce symptoms so mild that they remain undiagnosed. These mild infections are characterized by a general malaise, headache and a light fever.

For the $20 \%$ of cases where Lassa fever become serous, symptoms can include:

A. Hemorrhaging- in the gums, nose, eyes or elsewhere.

B. Difficulty breathing, cough, swollen airways, stomachache, vomiting and diarrhea (both bloody), difficulty swallowing, Hepatitis.

\section{Prevention and Control}

Given that the host of the Lassa virus is rodents which are so abundant in endemic areas, one plausible means of prevention is promoting good personal and community hygiene to discourage rodents from entering homes. According to the [6]. Practical examples here would include storing grain and other foodstuffs in rodent-proof containers, disposing of garbage far from the home, maintaining clean households, keeping cats to hunt vermin, cooking all foods thoroughly and constant hand washing [6]. More so, family members and health care workers should always be careful to avoid contact with blood and body fluids while caring for sick persons. Along this line, for added safety, patients suspected to have Lassa fever should be cared for under specific isolation precautions, which include the wearing of protective clothing such as masks, gloves, gowns and face shield and the systematic sterilization of contaminated equipment $[7,8]$.

\section{Conclusion}

This essay presents a succinct synopsis on the Lassa fever. The essay contends that the disease is only endemic in the Western, Central and Eastern part of Africa. It goes without saying that people in the western world are also susceptible since secondary transmission can occur by coming in contact with infected person's bodily fluid. As argued in the essay however, the disease is endemic in the Western, Central and Eastern Africa because Lassa virus the causative agent of the disease - is hosted in multi-mammate rat which is a common rodent in the region. Most important, these rodents live in most homes in the region and are even considered a delicacy. The study also points to the asymptomatic nature of the disease which in combination of the disease having some similar patterns with febrile illness and other RNA virus caused diseases makes diagnosis based on symptoms relatively impossible. Hence, properly designed clinical diagnosis like ELISA has been deemed a panacea. With respect to prevention, luck with clinical drugs or methods are scarce besides maintaining good personal and community hygiene to prevent the disease host reservoir Mastomys natalensis, from co-habiting with humans. In addendum, people should avoid eating food that has been contaminated with the rodent's faeces and urine. For treatment, only Ribavirin combined with supported care has been clinically approved. More so, the potency.

\section{References}

1. Sogoba N, Feldmann H, Safronetz D (2012) Lassa fever in West Africa: Evidence for an Expanded Region of Endemicity. Zoonoses \& Public Health 59: 43-47.

2. Donaldson, Ross I (2009) The lassa ward: One Mans's Fight Against One of the World Deadliest Diseases. Saint Martin's Press 24: 45-55.

3. Frame JD (2012) Surveillance of Lassa fever in missionaries stationed West Africa. Bull WHO 52(4-6): 593-598.

4. Gary GW (1977) A neutralization test survey for Lassa fever activity in Lassa, Nigeria. Transmission Routes Social Tropical Medical Hygiene 71(2): 152-154

5. Henderson BE (1972) Lassa fever: epidemiological aspects of the 1970 epidemic Jos, Nigeria. Transmission Routes Social Tropical Medical Hygiene 66(3): 402-408.

6. WHO (2017) Lassa fever fact sheet.

7. Buckley SM, Casals J, Downs WG (1970) Isolation and antigenic characterization Lassa virus. Nature 227:174-176.

8. Bowen GS, Tomori O, Wuff H, Casals J, Noonan A, et al. (1975) Lassa Fever in Onitsha, East Central State, Nigeria, Bull WHO 52(4): 559-604. 
This work is licensed under Creative Commons Attribution 4.0 License

To Submit Your Article Click Here:

Submit Article

DOI: 10.32474/IPDOAJ.2018.01.000116

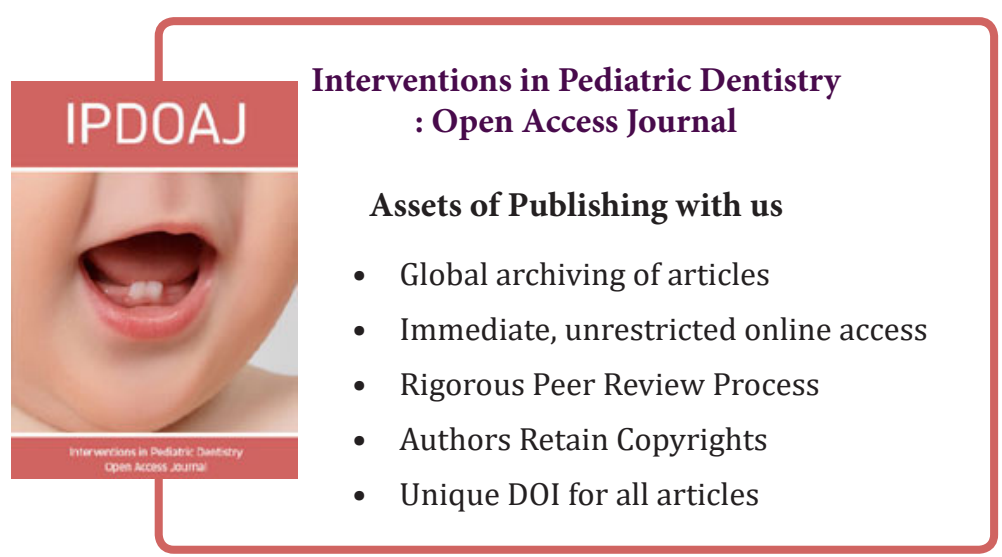

